\title{
Strategic Rail Network Optimization Model for Freight Transportation
}

\author{
Luís Couto Maia and António Couto
}

An optimization model for a rail freight network was developed as a support tool for planning and policy decisions involved in the improvement of rail networks on a regional and national level. The model was based on a strategic traffic assignment model, which was designed to model macronetworks with a high aggregation level, and was exclusively designed for freight traffic. The model contemplates road and rail transport modes and considers two types of cargo: intermodal cargo, which is generally transported in containers and is easily interchanged between different modes at intermodal terminals, and general cargo, which represents all the remaining cargo. The optimization process was based on a local search heuristic that delivers good solutions in a reasonable computing time. The quality of each network improvement solution was assessed on the basis of the reductions in total generalized costs and carbon dioxide emissions. This optimization model for a freight network is innovative because it is not limited: both the improvement of existing links and the construction of new ones are allowed, and the model does not have a limit on the number or variety of possibilities for network improvement. The adaptability to different conditions was emphasized when the model was applied to two networks under different investment scenarios because the model delivered considerably different solutions adapted to the conditions of each scenario.

Although freight transportation is an activity that plays a crucial role in the everyday life of any modern economy, being critical to a large part of the economy, it usually gets less attention in academia than its counterpart, passenger transportation. This is probably justified not only by the fact that it is not as appealing to policy makers and the general public as passenger transportation but also because it is a considerably more complex subject because of the multiplicity of goods transported, the complexity of the freight supply chain, and the difficulty with the collection of the needed data.

Although freight transportation may be less appealing and more complex than passenger transportation, it is important to study freight transportation through the use of models specifically made for such studies to account for its distinct characteristics and for the fact that the network investments needed to improve freight transportation can be considerably different from those needed to improve passenger transportation. For this reason, the network optimization model and the associated traffic assignment model that are presented in this paper have been developed specifically for this type of transportation,

Civil Engineering Department, Transport Infrastructure Division, University of Porto, Rua Dr. Roberto Frias, s/n, 4200-465 Porto, Portugal. Corresponding author: L. C. Maia, luis.maia@fe.up.pt.

Transportation Research Record: Journal of the Transportation Research Board, No. 2378, Transportation Research Board of the National Academies, Washington, D.C., 2013, pp. 1-12.

DOI: $10.3141 / 2378-01$ although they may be combined with passenger transportation models in the future to create a model for the whole transportation system.

The model presented here, developed in the scope of a broader project (1), uses a strategic planning traffic assignment model designed to model macronetworks with a high level of aggregation (2). This assignment model does not require detailed data inputs; and the outcome of its application is an estimate of the movement of freight at a regional, national, or international scale. It considers road and rail transport modes and is intended to simulate medium- and long-distance flows of inland transportation. The model contemplates two types of cargo, namely, $(a)$ general cargo and intermodal cargo, to make a distinction between the cargo that may be easily interchanged between different modes at intermodal terminals, which is generally transported in containers, and $(b)$ all remaining cargo. The characteristics described above make this traffic assignment model particularly suited for the planning and policy decisions that will be performed by the network optimization model (3).

The optimization process itself is flexible and innovative and allows upgrades to both the quality of existing rail and intermodal terminal links and the construction of new ones, with no limit on the number or variety of improvement solutions. This possibility for unlimited improvements is achieved by definition of a set of possible link levels for each link type, according to the users' preferences, including the mere possibility of building of a link. The quality of each network improvement solution is assessed on the basis of the reduction of total generalized costs and carbon dioxide $\left(\mathrm{CO}_{2}\right)$ emissions, with the weights given to each of those parameters defined by the user according to the user's preferences. The optimization model is based on a search heuristic and tries to meet a balance between efficiency and effectiveness by delivery of good solutions in a reasonable computing time.

This paper is structured in six sections. After this introduction, a background section contains a brief literature review on the subject of freight traffic assignment and network optimization models. The following sections are dedicated to the traffic assignment model and the network optimization process. An application of the network optimization model is then described, and the last section is dedicated to the final conclusions.

\section{BACKGROUND}

\section{Models for Freight Traffic Assignment}

The different traffic assignment techniques that are presented in the literature can be divided into four groups: all-or-nothing, equilibrium, stochastic-multiflow, and stochastic-equilibrium (4). The two factors that determine to which of the four groups a model belongs are capacity constraints imposed by congestion (equilibrium and 